\title{
Aplicativo de Aprendizagem Móvel utilizando Realidade Aumentada para Ensino de Língua Inglesa
}

\author{
Rafael Costa de Oliveira1, Danila Silva1, Flávia Gonçalves Fernandes², \\ Luciene Chagas de Oliveira ${ }^{1,2}$, Eduardo Chagas de Oliveira ${ }^{1,2}$ \\ ${ }^{1}$ Universidade de Uberaba (UNIUBE) - Uberlândia, MG - Brasil \\ ${ }^{1}$ Universidade Federal de Uberlândia (UFU) - Uberlândia, MG - Brasil \\ rafa_costa_ @hotmail.com, danilasistemas@gmail.com, \\ flavia. fernandes92@gmail.com, lchagasoliveira@gmail.com, \\ edu_ufudyahoo.com.br
}

\begin{abstract}
The Augmented Reality (AR) can be a great tool in education. Currently, many of the students along the way lose the essence and importance is the study and pursuit of knowledge. It is therefore necessary to seek something different, something that is truly innovative to encourage them to study more. Given this, there is the idea of building interactive software, oriented education. This paper shows the contribution of AR technology for education and development of an implementation case study for mobile learning foreign language.

Resumo. A Realidade Aumentada (RA) pode ser uma grande ferramenta na área da educação. Atualmente, muitos alunos perdem ao longo do caminho a essência e a importância do estudo e a busca pelo conhecimento. Por isso, é necessário buscar algo diferente, algo que seja realmente inovador para incentivá-los a estudar mais. Diante nisso, surge a ideia de se construir softwares interativos, voltados para área da educação. Este artigo apresenta a contribuição da tecnologia de RA para a educação e o desenvolvimento de uma aplicação de estudo de caso para aprendizagem móvel da língua inglesa.
\end{abstract}

\section{Introdução}

As inovações tecnológicas têm atuado de forma significativa na área da educação, quebrando velhos paradigmas na aprendizagem dos alunos, baseando nas práticas pedagógicas de ensino que visa estimular a curiosidade e desenvolvimento do conhecimento, afirmando ao aluno a competência que a prática do saber agrega valores nas perspectivas futuras. A partir desse conceito, dentre várias opções na área tecnológica, pode-se citar que a Realidade Aumentada (RA) tem sido bastante utilizada para o desenvolvimento do conhecimento, tornando prática e dinâmica a interatividade do usuário juntamente com a participação da mobilidade oferecida pelos dispositivos móveis, tais como, smatphones e tablets, no desenvolvimento do conhecimento [Tori, 2006].

Os principais benefícios do uso de dispositivos móveis para a aprendizagem incluem: a portabilidade, a mobilidade: estar disponível a qualquer hora e em qualquer lugar, acesso de forma flexível a diversos recursos e em tempo real, economia de tempo, rapidez da comunicação, capacitação e envolvimento dos alunos e o fato da aprendizagem móvel (mobile learning) ser abrangente e dinâmica [Woodill, 2010].

De acordo com um relatório da ONU, seis em cada dez pessoas no mundo têm assinatura de telefonia móvel, o que significa que os celulares representam a 
comunicação tecnológica escolhida nos últimos anos, especialmente nos países mais pobres [Woodill, 2010].

Alguns fatores de mercado contribuem para efetivação de aplicativos e softwares com a RA, como a diminuição do custo dos equipamentos que possui tecnologias adequadas para o desenvolvimento e utilização desses aplicativos, se tornam cada vez mais acessíveis nas diversas classes sociais.

A amplitude em que a RA vem atuando se tornar imprescindível e coerente para várias soluções contribuindo para o desenvolvimento social e tecnológico.

Atualmente, vem crescendo a quantidade de ferramentas e aplicativos educativos que remetem alunos, professores e profissionais, sejam eles tecnológicos ou educacionais, a se tornarem usuários e desenvolvedores de aplicações cada vez mais eficientes e eficazes na educação. Com módulos básicos e avançados, nas áreas de humanas, exatas e ciências naturais, o aluno se dispõe de conteúdo ilustrativo e principalmente interativo em jogos e livros em formato tridimensional [Tori, 2006].

Utilizando de um método educacional articulado que visa estimular a interação entre aluno e tecnologia em tempo real, com formas e figuras tridimensionais, combinando objetos reais e virtuais, em um âmbito qualitativo esse método de conhecimento permite uma forma organizada de ensino [Milgram, 1994].

A importância desse estudo visa utilizar das metodologias de ensino e aprendizagem, e a contribuição da tecnologia como forma de estimulo de ensino, baseando-se em promover conhecimento, a partir da mobilidade, dinamismo e usabilidade de um aplicativo que remete a sensação de interação do mundo real com o virtual [Milgram, 1994].

Neste sentido, este trabalho tem como objetivo apresentar uma aplicação da Realidade Aumentada para aprendizagem móvel da língua inglesa.

\section{A Contribuição da Realidade Aumentada na Aprendizagem Móvel}

A Aprendizagem Móvel pode ser definida como qualquer disposição de ensino onde as tecnologias dominantes são os dispositivos portáteis [Kukulska-Hulme, 2005].

Aprendizagem móvel é agora visto como um meio de manter as pessoas em contato com entre si e com as fontes de informação, independentemente de onde quer que esteja localizado [Woodill, 2010].

A educação é o campo que nos interessa, é estruturada em competências e em capacidade de oferecer ao sujeito a se mobilizar diante de uma diversidade de recursos na solução e descobertas de novas vertentes ou já existentes para seu desenvolvimento social e cultural.

$\mathrm{Na}$ agregação de valores e conhecimento, a educação tem se apoiado as inovações tecnológicas e a disposição de recursos que visam explorar o conhecimento de forma curiosa e intuitiva a partir de jogos e aplicativos que utilizam da RA.

$\mathrm{Na}$ restruturação das formas de aprendizagem baseando-se nos princípios socioculturais, professores, pedagogos e desenvolvedores de tecnologias, com o objetivo de observar, regular e inovar, visam explorar o conhecimento utilizando das inovações 
tecnológicas que tem a capacidade de envolver e despertar o interesse de aprendizagem onde o aluno se torna construtor do seu próprio conhecimento. Sendo assim, o usuário deixa de ser um receptor de informações para ser um elaborador de representações [Luckesi, 1995].

A mobilidade está presente onde o aluno estiver e os dispositivos móveis são tecnologias que auxiliam na criação de experiências de aprendizagem. Atividades como jogos ao ar livre, passeios de museus e galerias, e o uso da Realidade Aumentada para complementar as informações em cada local são algumas das atividades que melhor se adequam a este tipo de aprendizagem móvel [Woodill, 2010].

A partir dessas e outras sobreposições, é possível avaliar de forma positiva a capacidade e a competências da utilização da RA e suas aplicações, que oferece conhecimento, a instrução e profissionalismos de alunos e usuários que alcançam o sucesso e superam suas próprias expectativas de entendimento.

Dentre várias vantagens da RA encontram-se: a motivação do estudante, a interação em as disposições multimídia podendo ver de forma tridimensional figuras, remete o aluno sair do âmbito da sala de aula e oferece a capacidade de se colocar dentro do mundo virtual, a criatividade, o desenvolvimento cultural, e a habilidade e o domínio nas técnicas computacionais.

A RA vem beneficiando várias áreas, abrangendo o conhecimento básico ao avançado. Experiências de utilização de sistemas que utilizam de técnicas de Realidade Aumentada têm sido desenvolvidas e aplicadas em diversos campos de ensino, desde Medicina, indústria e Educação, tais como aplicativos de matemática básica, experimentos virtuais de Óptica Geométrica e até simulações de circuitos integrados [Cardoso, 2001].

Portanto, dentre os fatores abordados, é importante a constância no trabalho de desenvolver, aperfeiçoar novas e já existentes técnicas de aprendizagem que otimiza e capacita o desenvolvimento social e cultural, estimulando a arte de aprendizagem.

\section{Trabalhos Relacionados}

Nesta seção, são descritos alguns trabalhos relacionados ao ambiente virtual e sua contribuição na educação.

A partir da análise dos trabalhos foram levantados os seguintes requisitos para a desenvolvimento do software, são eles: a utilização da mobilidade (tablet $e$ smartphones); a utilização de sistema operacional específico e a aprendizagem da língua inglesa.

O Jogo de Palavras proposto em [Kirner, 2005] que utiliza da Realidade Aumentada tem como objetivo fazer a junção de letras para formar palavras e resgatando a imagem referente resultante a palavras formada com a técnica da RA para enriquecer os resultados finais. Neste trabalho foi utilizado o ARToolkit [Azuma, 1993] que é um software livre bastante utilizado para o desenvolvimento de aplicações de RA.

A Figura 1 apresenta alguns modelos de placas cadastradas junto aos seus respectivos objetos virtuais, usando da palavra em inglês. 


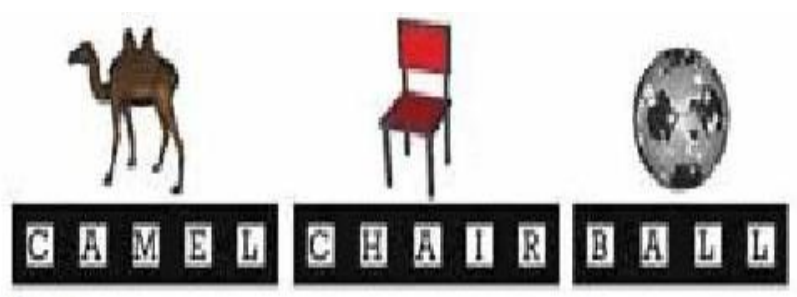

Figura 1: Exemplo de placas objetos virtuais correspondentes [Kirner, 2005]

Outro trabalho é o jogo de quebra-cabeças educacional proposto em [Azuma, 1993], que discute o uso da tecnologia de RA no desenvolvimento de quebra-cabeças tridimensionais, visualizados com a ajuda do computador, mostrando que é possível através de uma plataforma computacional simples e software gratuito, criar quebracabeças eletrônicos enriquecidos, motivadores e de fácil usabilidade, conforme ilustrado na Figura 2.

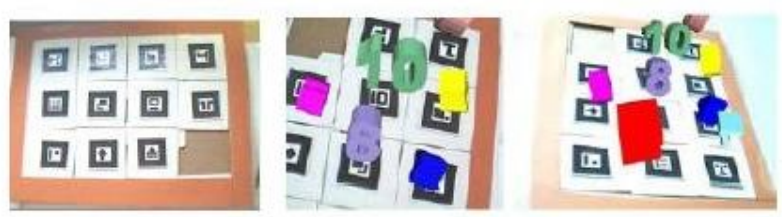

Figura 2: Quebra-Cabeça [Azuma, 1993].

Os trabalhos de aprendizagem apresentados nesta seção não utilizam dispositivos móveis para o uso da RA.

\section{Aplicação Móvel de Aprendizagem de Língua Inglesa com RA}

Diante dos trabalhos encontrados, o aplicativo What Is Figure? agrega a tecnologia da mobilidade propiciando a prática de aprendizagem em qualquer lugar a qualquer momento.

Para o desenvolvimento da ferramenta, foi utilizado o conceito da visão por vídeo ou tela do dispositivo móvel através da captura da imagem a partir da câmera do aparelho apontada no marcador. O marcador é predefinido no sistema que reconhece as imagens retornando o resultado a partir da escolha realizada pelo usuário no menu inicial do aplicativo.

A biblioteca de software utilizada para o desenvolvimento do aplicativo foi a AndAR (Android Augmented Reality) que implementa Java baseado em Android e utiliza o ARToolkit para reconhecer os marcadores e imagens através de sobreposições de vídeo ao vivo do mundo real [Project, 2015].

A plataforma Android [Lecheta, 2015] atualmente é tecnologia predominante nos dispositivos moveis e de baixo custo que vem ganhando grande espaço no mercado tecnológico. Também compõem este aplicativo as imagens tridimensionais criadas e desenvolvidas na plataforma Blender que dispõe de uma variedade ferramentas para manipular e criar imagens em 3D com contornos e perfeitos [Blender, 2015]. 
V Congresso Brasileiro de Informática na Educação (CBIE 2016)

Anais dos Workshops do V Congresso Brasileiro de Informática na Educação (CBIE 2016)

Neste trabalho foi desenvolvido uma aplicação, denominada What is Figure?, para aprendizagem móvel da língua inglesa utilizando RA. Esse sistema tem como objetivo ajudar na assimilação de palavras da língua inglesa relacionando-as com figuras que são apresentadas no mundo real, no formato tridimensionais, utilizando RA e dispositivos móveis. Essa didática visa conhecimento básico da língua inglesa, onde a interação entre mundo real e virtual estará disponível em um serviço móvel tonando mais disponível o conhecimento ao usuário.

Na Figura 3, é apresentado o diagrama de casos de uso da aplicação.

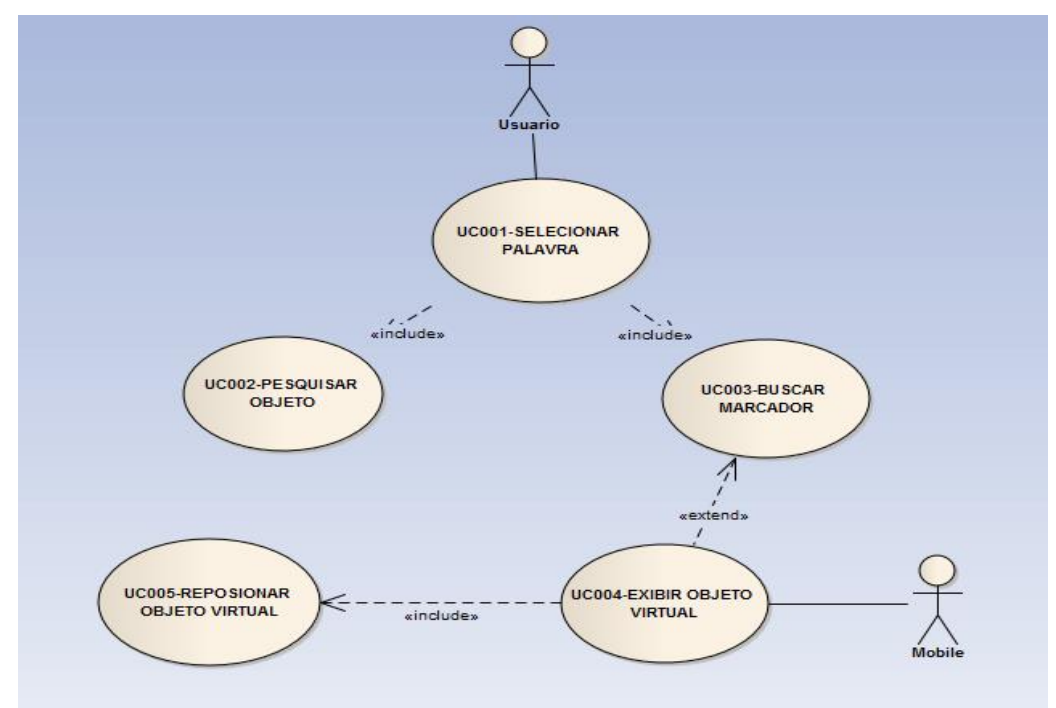

Figura 3: Diagrama de casos de uso da aplicação.

O sistema funciona da seguinte maneira: Primeiramente, é necessário instalá-lo em um celular e, em seguida, procurar e selecionar o aplicativo "What Figute Model View" entre os aplicativos do celular, conforme mostra a Figura 4.

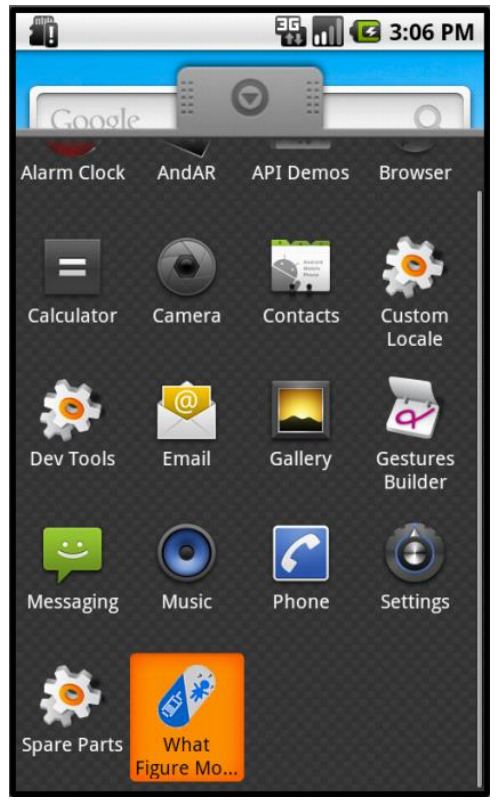

Figura 4: Seleção do sistema entre os aplicativos do celular. 
V Congresso Brasileiro de Informática na Educação (CBIE 2016)

Anais dos Workshops do V Congresso Brasileiro de Informática na Educação (CBIE 2016)

A aplicação em execução é apresentada na Figura 5.
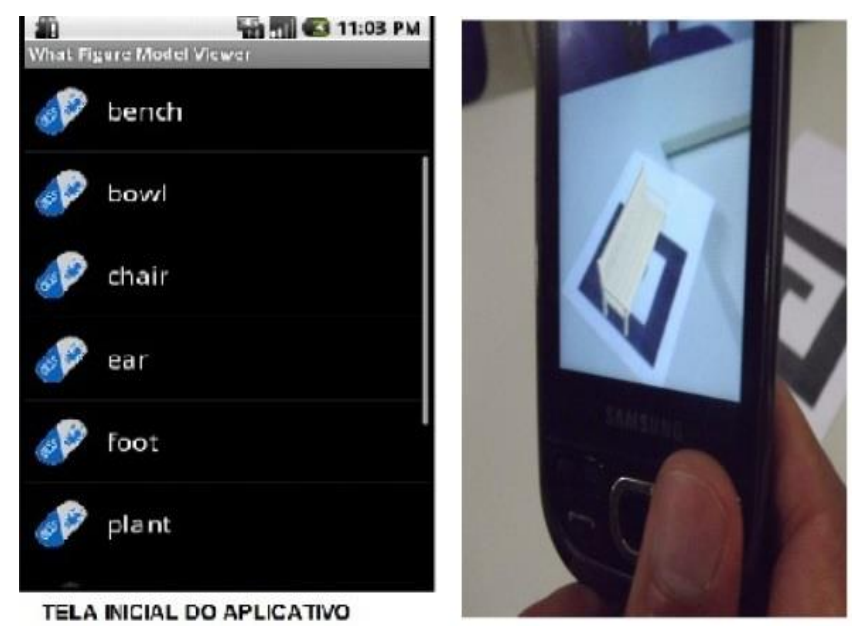

Figura 5: Menu inicial e visualização do usuário utilizando RA.

A Figura 6 apresenta a tela inicial da aplicação, onde são apresentados para o usuário uma lista de palavras do idioma inglês. O usuário seleciona a palavra que deseja visualizá-la em forma de um objeto virtual. Em seguida, é apresentada uma lista que contém todas as palavras disponibilizadas pelo sistema.

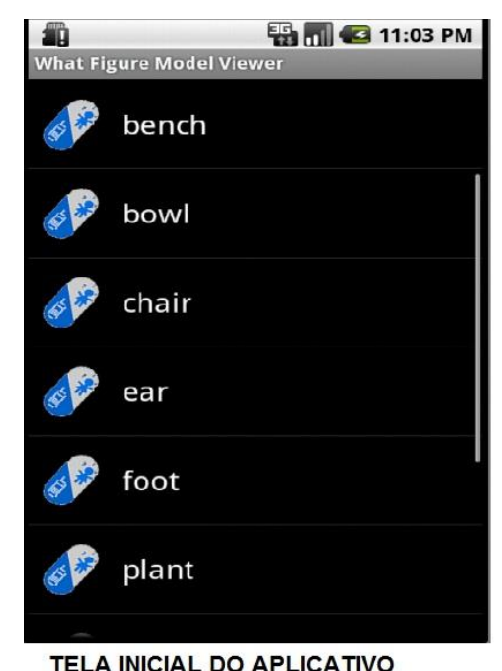

Figura 6: Tela contendo as palavras na língua inglesa.

A Figura 7 apresenta a seleção de uma palavra em língua inglesa, que ocorre ao clicar sobre a mesma no aplicativo do celular. 
V Congresso Brasileiro de Informática na Educação (CBIE 2016)

Anais dos Workshops do V Congresso Brasileiro de Informática na Educação (CBIE 2016)

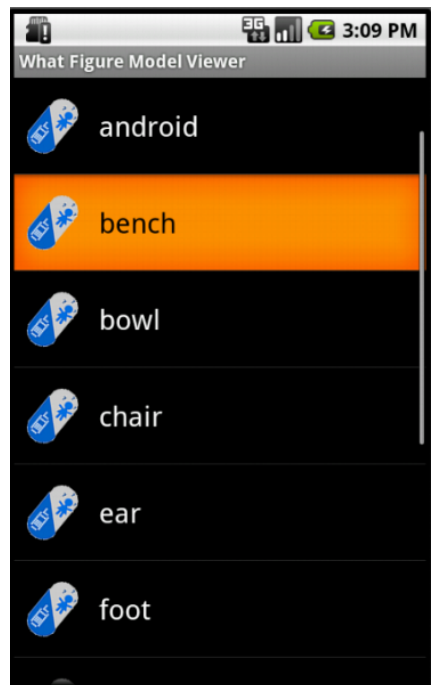

Figura 7: Seleção de uma palavra em língua inglesa.

Na Figura 8, encontra-se uma tela de carregamento do objeto referente à palavra selecionada, contendo uma mensagem de aviso ao usuário sobre esse processo.

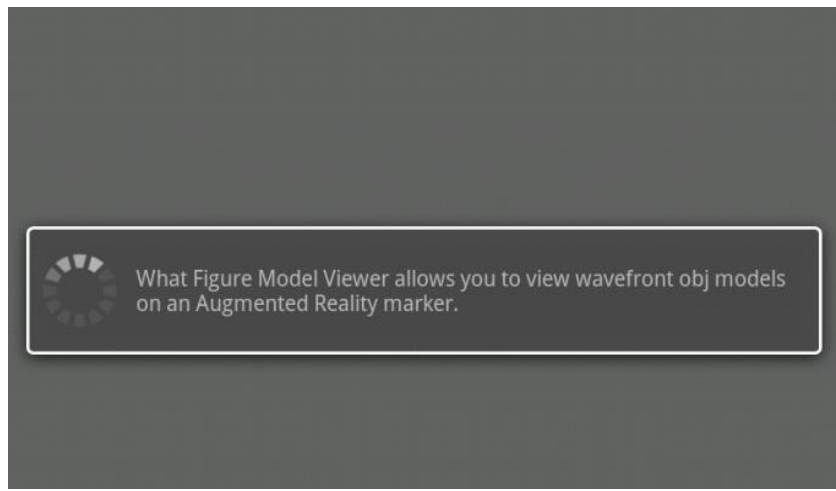

Figura 8: Carregamento do objeto selecionado.

$\mathrm{Na}$ Figura 9, é apresentado o marcador utilizado para a execução dessa aplicação.

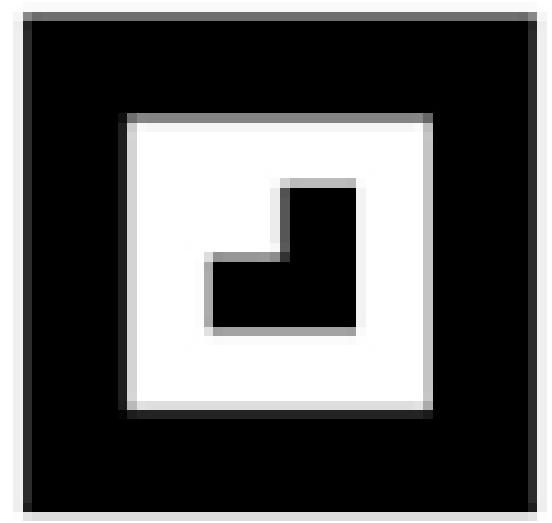

Figura 9: Marcador utilizado na aplicação. 
V Congresso Brasileiro de Informática na Educação (CBIE 2016)

Anais dos Workshops do V Congresso Brasileiro de Informática na Educação (CBIE 2016)

Em seguida, o usuário deve posicionar a câmera do celular sobre o marcador fiducial para que o objeto tridimensional referente à palavra selecionada seja exibido em Realidade Aumentada, como pode ser visto nas Figuras 10, 11 e 12.

$\mathrm{Na}$ Figura 10, é apresentado um banco (bench em inglês) em Realidade Aumentada pelo celular, após posicionar a câmera sobre o marcador.

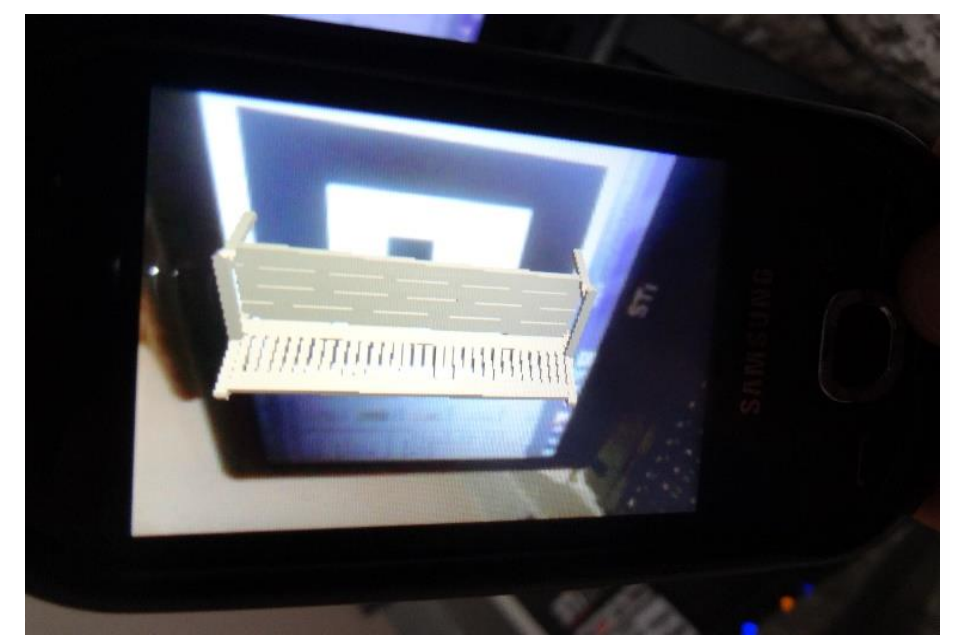

Figura 10: Exibição em RA de um banco após seleção da palavra bench.

Na Figura 11, é apresentado um androide (android em inglês) em Realidade Aumentada pelo celular, após posicionar a câmera sobre o marcador.

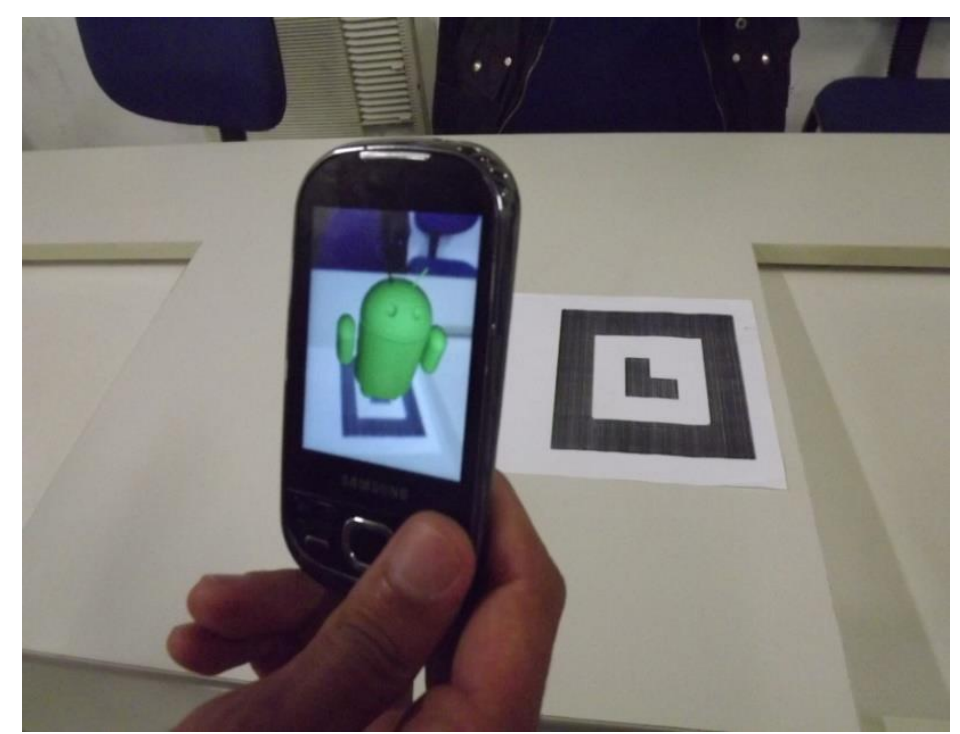

Figura 11: Exibição em RA de um androide após seleção da palavra android.

$\mathrm{Na}$ Figura 12, é apresentado um sorvete (ice em inglês) em Realidade Aumentada pelo celular, após posicionar a câmera sobre o marcador. 


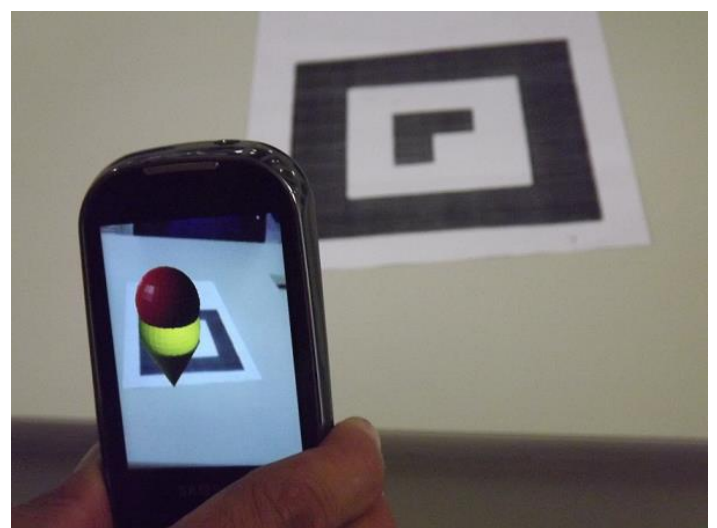

Figura 12: Exibição em RA de um sorvete após seleção da palavra ice.

Após a conclusão de desenvolvimento do aplicativo, pode-se analisar as necessidades de melhoramento para trabalhos futuros, além disso, pode-se destacar já a importância da utilidade do aplicativo no desenvolvimento do conhecimento de crianças e até mesmo de adultos em um nível básico para aprender e desenvolver a língua inglesa. A experiência de teste foi realizada entre os desenvolvedores e usuário final.

Diante das figuras desenvolvidas, a ferramenta possui imagens do cotidiano, a assimilação da imagem e a palavra é a combinação perfeita para o entendimento do usuário.

O sistema consegue reconhecer o marcador a partir de qualquer superfície plana ou não com ambiente de luminosidade clara ou natural.

Para utilizar o aplicativo, o usuário deverá possuir um dispositivo móvel que possuam o sistema operacional Android e instalar o aplicativo no aparelho.

\section{Conclusões e Trabalhos Futuros}

Pode-se notar a expansão que a Realidade Aumentada tem na implementação de ferramentas e sua contribuição no desenvolvimento de aplicativos na área da Educação.

A partir de suas características de usabilidade, dinamismo, capacidade de promover a interação entre os mundos real e virtual, torna a capacidade de aprender e ensinar fascinante diante da disposição de se ter o conhecimento no cotidiano em qualquer lugar.

A carência de estimulo a educação, promove o desenvolvimento e criação de aplicativos que rompam as barreiras que impedem o desenvolvimento social e tecnológico.

Entretanto, o estímulo de se ter a tecnologia a disposição da Educação em qualquer lugar a qualquer momento ajuda a prática dos estudos e da busca por novos conhecimentos, auxiliando na capacidade de entendimento do usuário ou aluno diante da ferramenta, de forma didática e objetiva. 
V Congresso Brasileiro de Informática na Educação (CBIE 2016)

Anais dos Workshops do V Congresso Brasileiro de Informática na Educação (CBIE 2016)

Com isso, este trabalho discutiu a utilização da tecnologia de Realidade Aumentada combinada a aprendizagem móvel para criação de ambientes de ensino permitindo ampliar as possibilidades pedagógicas.

A aplicação desenvolvida propõe minimizar as dificuldades de entendimento de alunos diante da língua inglesa, com o auxílio da tecnologia RA móvel, de forma a tornar estimulante a arte da aprendizagem.

Como proposta de melhorias futuras propõe-se adicionar novas funcionalidades na aplicação, tais como, a busca por palavras e a escolha de outras línguas inglesas.

\section{Referências}

Azuma, R. M. Billinghurst and G.. Klinker. 2011. "Editorial: Special Section on Mobile Augmented Reality", in Computer Graphics. pp. vii-viii.

Azuma, R. T. Tracking Requirements for Augmented Reality, Communications of the ACM, 36(7):50-51, July 1993.

Blender. 2015, [Online; accessed 20-Set-2015]. [Online]. Available: http://www.blender.org/

Cardoso, A.; Lamounier Jr. And Tori, R. 2001."Interactive 3D Physics Experiments through the Internet", Proceedings of the 4th SBC Symposium on Virtual Reality,pp.280-90, Florianópolis,SantaCatarina, Brazil. http://users.hub.ofthe.net/ mtalkmit/veshtml2.html, November.

Kirner, C; Zorzal, E. "Jogos Educacionais em Ambiente de Realidade Aumentada". Workshop de Realidade Aumentada, PiracIcaba- São Paulo, 2005.

Kukulska-Hulme, A., and Traxler, J. (eds.) (2005). "Mobile Learning: A Handbook for Educators and Trainers". London: Routledge.

Lecheta, R. R. Google Android, 2nd ed., Novatec, Ed., 2015. [Online]. Available: http://www.livroandroid.com.br/.

Luckesi, C. C. “Avaliação da aprendizagem escolar: estudos e proposições”. São Paulo: Cortez, 1995.

Milgram, P. et. al. (1994) "Augmented Reality: A Class of Displays on the RealityVirtuality Continuum”. Telemanipulator and Telepresence Technologies, SPIE, V.2351, p. 282-292.

Project, G. "Andar - android augmented reality". 2015. [Online]. Available: http://code.google.com/p/andar/.

Tori, R.; Kirner, C.; Siscouto, R...'Fundamentos e Tecnologia de Realidade Virtual e Aumentada". Belém: VIII Symposium On Virtual Reality, 2006.

Woodill, G. "The Mobile Learning Edge: Tools and Technologies for Developing Your Teams", 1st ed., McGraw-Hill, Ed., 2010. [Online]. Available: http://www.mobilelearningedge.com. 